\title{
High prevalence of asymptomatic malaria infections in adults, Ashanti Region, Ghana, 2018
}

\author{
Melina Heinemann ${ }^{1,2,8 \dagger}$, Richard O. Phillips ${ }^{3 \dagger}$, Christof D. Vinnemeier ${ }^{2,4}$, Christina C. Rolling ${ }^{4,9}$, Egbert Tannich ${ }^{5,6+}$ \\ and Thierry Rolling $1,6,7,10^{*}+$ (D)
}

\begin{abstract}
Background: Ghana is among the high-burden countries for malaria infections and recently reported a notable increase in malaria cases. While asymptomatic parasitaemia is increasingly recognized as a hurdle for malaria elimination, studies on asymptomatic malaria are scarce, and usually focus on children and on non-falciparum species. The present study aims to assess the prevalence of asymptomatic Plasmodium falciparum and non-falciparum infections in Ghanaian adults in the Ashanti region during the high transmission season.
\end{abstract}

Methods: Asymptomatic adult residents from five villages in the Ashanti Region, Ghana, were screened for Plasmodium species by rapid diagnostic test (RDT) and polymerase chain reaction (PCR) during the rainy season. Samples tested positive were subtyped using species-specific real-time PCR. For all Plasmodium ovale infections additional subspecies identification was performed.

Results: Molecular prevalence of asymptomatic Plasmodium infection was 284/391 (73\%); only 126 (32\%) infections were detected by RDT. While 266 (68\%) participants were infected with Plasmodium falciparum, 33 (8\%) were infected with Plasmodium malariae and 34 (9\%) with P. ovale. The sub-species P. ovale curtisi and P. ovale wallikeri were identified to similar proportions. Non-falciparum infections usually presented as mixed infections with P. falciparum.

Conclusions: Most adult residents in the Ghanaian forest zone are asymptomatic Plasmodium carriers. The high Plasmodium prevalence not detected by RDT in adults highlights that malaria eradication efforts must target all members of the population. Beneath Plasmodium falciparum, screening and treatment must also include infections with $P$. malariae, P. o. curtisi and P. o. wallikeri.

Keywords: Asymptomatic malaria, Plasmodium falciparum, Plasmodium malariae, Plasmodium ovale curtisi, Plasmodium ovale wallikeri, Ghana, Sub-Saharan Africa, Rapid diagnostic test, Polymerase chain reaction, Molecular prevalence

\footnotetext{
*Correspondence: rollingt@mskcc.org

${ }^{\dagger}$ Melina Heinemann and Richard O. Phillips-first authors contributed equally to this article

†Egbert Tannich and Thierry Rolling — senior authors contributed equally to this article

${ }^{1}$ Division of Infectious Diseases, I. Department of Medicine, University Medical Centre Hamburg-Eppendorf, Hamburg, Germany

Full list of author information is available at the end of the article
}

\section{Background}

The World Health Organization (WHO) aims to achieve a global reduction in malaria case incidence and mortality rates by $90 \%$ compared to 2015 , elimination in at least 35 countries, and prevention of reintroduction in all malaria-free countries by the year 2030. Although the worldwide burden of malaria substantially decreased since 2010, some high-burden countries in Africa report 
an increase in malaria cases. According to the WHO, the global targets for 2030 will not be achieved unless there is accelerated change. Ghana belongs to the eleven high-burden countries accounting for $>70 \%$ of the global malaria cases and deaths. With an increase of malaria cases by $8 \%$, Ghana is among the two highest burden countries in Africa reporting the highest absolute increase in malaria cases in 2018 compared to 2017 [1].

Targeting malaria elimination, mass screening and treatment (MSAT) aims to detect and treat all infections, including asymptomatic Plasmodium carriers with low-density infection, to reduce the parasite reservoir [2, 3]. MSAT usually uses a rapid diagnostic test (RDT) as diagnostic tool. RDTs have a sensitivity comparable to field microscopy. While RDTs are limited regarding sensitivity for Plasmodium falciparum infections with $<100$ parasites/ $\mu$ l and non-falciparum infections [2], polymerase chain reaction (PCR) has a limit of detection as low as 0.02 parasites $/ \mu$ l and enables differentiation of nonfalciparum species [2, 4]. Since the proportion of submicroscopic malaria infections depends on the transmission intensity and ranges from 20 to $80 \%$, molecular diagnostic methods are necessary to understand local malaria epidemiology [3].

Despite asymptomatic parasitaemia being increasingly recognized as a major hurdle towards malaria eradication, it has rarely been a major research focus. Especially studies on asymptomatic adults and asymptomatic nonfalciparum infections are scarce $[5,6]$.

The present work reports the results of a survey using both RDT and PCR to assess asymptomatic carriage of $P$. falciparum and non-falciparum species in adult residents of a Ghanaian forest region during the high transmission season in order to inform policy-makers regarding the optimal strategies to reduce malaria burden in Ghana.

\section{Methods}

Adults volunteers were recruited during the second rainy season in September 2018 in five villages in the Asante Akim North district in the Ashanti region, Ghana. The district lies within the moist semi-deciduous forest belt of Ghana. The forest areas are characterized by a tropical climate with two rainy seasons [7]. The villagers live in simple houses constructed from local materials and their main occupation is farming. One to three days before recruitment, community health workers visited the representative villages and invited all adults who lived in the catchment area to participate. Exclusion criteria were clinical signs of infection (axillary temperature $\geq 37.5{ }^{\circ} \mathrm{C}$ or history of fever in the past $48 \mathrm{~h}$, headache, chills, myalgia, dizziness, nausea and diarrhoea), pregnancy and puerperium.
On the day of blood collection, field workers informed the villagers again about the exclusion criteria and the planned procedure of blood collection. Participants were only included after providing written informed consent. Age, gender and absence of exclusion criteria were assessed with a questionnaire for each participant. An RDT targeting the histidine-rich protein II antigen specific to $P$. falciparum and a pan-malarial antigen common to Plasmodium vivax, Plasmodium ovale, and Plasmodium malariae was performed on venous blood immediately to inform all participants about their test result (BinaxNOW Malaria Test; Binax, Inc., Scarborough, ME, USA). According to the WHO guideline, faint test bands were interpreted as positive [8]. Similar to a recent study, faint test bands were defined as being only visible in a good light in agreement of two members of the study team observing the test [9]. If the band was difficult to see in good light but both members of the study team still agreed to see it, the test was interpreted as very faint and reported separately.

For blood counts and PCR, venous blood was collected in EDTA blood tubes (Sarstedt). Blood counts were performed the same day using the Sysmex XP-300 automated hematology analyzer (Sysmex, Kobe, Japan). For PCR analyses EDTA-anticoagulated blood was centrifuged and the pellet was collected and stored at $-80^{\circ} \mathrm{C}$. Frozen samples were shipped to the National Reference Centre for Tropical Pathogens, Bernhard Nocht Institute of Tropical Medicine, Hamburg, Germany. Nucleic acid was extracted manually from $200 \mu \mathrm{l}$ of frozen red blood pellet using the QIAamp DNA Blood Mini-Kit (Qiagen, Hilden, Germany) according to the manufacturer's instructions. Screening was performed by genus-specific real-time PCR for Plasmodium species (RealStar Malaria PCR kit 1.0, Altona Diagnostics, Hamburg, Germany) as described before [10,11].

Positive samples were subtyped using species-specific real-time PCR targeting $P$. falciparum, $P$. vivax, $P$. ovale, $P$. malariae, and Plasmodium knowlesi (Altona Diagnostics, Hamburg, Germany) as described by the manufacturer and others $[10,11]$. If the commercial species-specific PCR was negative, a real-time in-house one-tube SybrGreen malaria PCR was performed as described previously. The species-specific in-house PCR targets $P$. falciparum, $P$. knowlesi, $P$. vivax, $P$. ovale, and $P$. malariae and was earlier shown to be more inhibition-resistant compared to the commercial PCR [11]. Furthermore, sub-species identification was performed for all $P$. ovale infections using an in-house PCR according to the protocol of Bauffe et al., as previously described [12]. Data analyses were conducted using R (R Foundation for Statistical Computing, version 3.4.3). 
The study was approved by the Committee on Human Research, Publication and Ethics at the Kwame Nkrumah University of Science and Technology, Kumasi, Ghana (CHRPE/AP/455/18).

\section{Results}

A total of 401 participants were screened for symptoms. One person was excluded from the study before blood collection due to dizziness. Therefore, 400 participants were included in the study. In 16 out of 400 samples both malaria PCR and internal control PCR (IC) were initially negative, indicating PCR inhibition. The samples were diluted 1:10 and measured again. After dilution, four of the 16 samples tested positive for Plasmodium species, three were negative and nine remained malaria PCR and IC negative and thus were excluded from the study.

The remaining 391 participants, including 206 women and 185 men, had a median age of 32 years (interquartile range [IQR] 25-44 years). The localization of the study sites in the Asante Akim North district and the range of participants with asymptomatic Plasmodium infection among the villages are shown in Fig. 1a, b. Village coordinates are given as previously reported [13]. A total of 284 study participants (73\%) tested positive for Plasmodium species by PCR. While $266(68 \%)$ participants tested positive for $P$. falciparum, 33 (8\%) tested positive for $P$. malariae and 34 (9\%) for P. ovale. (Table 1). Both P. ovale curtisi $(3 \%)$ and P. ovale wallikeri (4\%) were identified at
Table 1 Plasmodium species detection

\begin{tabular}{lrr}
\hline Plasmodium species detection & \multicolumn{2}{c}{ PCR (total $\mathbf{n = 3 9 1 )}$} \\
\cline { 2 - 3 } & No & $\%$ \\
\hline Pan-Plasmodium positive & 284 & 73 \\
P. falciparum & 266 & 68 \\
Monoinfection & 218 & 56 \\
Coinfection with P. malariae & 22 & 6 \\
Coinfection with P. ovale & 20 & 5 \\
Coinfection with P. malariae + P. ovale & 6 & 2 \\
P. malariae & 33 & 8 \\
Monoinfection & 5 & 1 \\
Coinfection & 28 & 7 \\
P. ovale & 34 & 9 \\
Monoinfection & 8 & 2 \\
Coinfection & 26 & 7 \\
Species not determined & 5 & 1 \\
\hline
\end{tabular}

$\mathrm{a} n=15(4 \%)$ P. ovale wallikeri, $\mathrm{n}=12$ (3\%) P. ovale curtisi, $\mathrm{n}=7$ (2\%) unknown subspecies

similar rates. In seven cases $(2 \%)$ with high cycle threshold $\left(C_{t}\right)$ values (low parasite density) sub-species differentiation was unsuccessful. All participants were negative for $P$. vivax and $P$. knowlesi.

While $P$. falciparum presented mainly as monoinfection (82\%), P. malaria and $P$. ovale were observed mainly in the context of coinfections with $85 \%(28 / 33)$ and $76 \%$

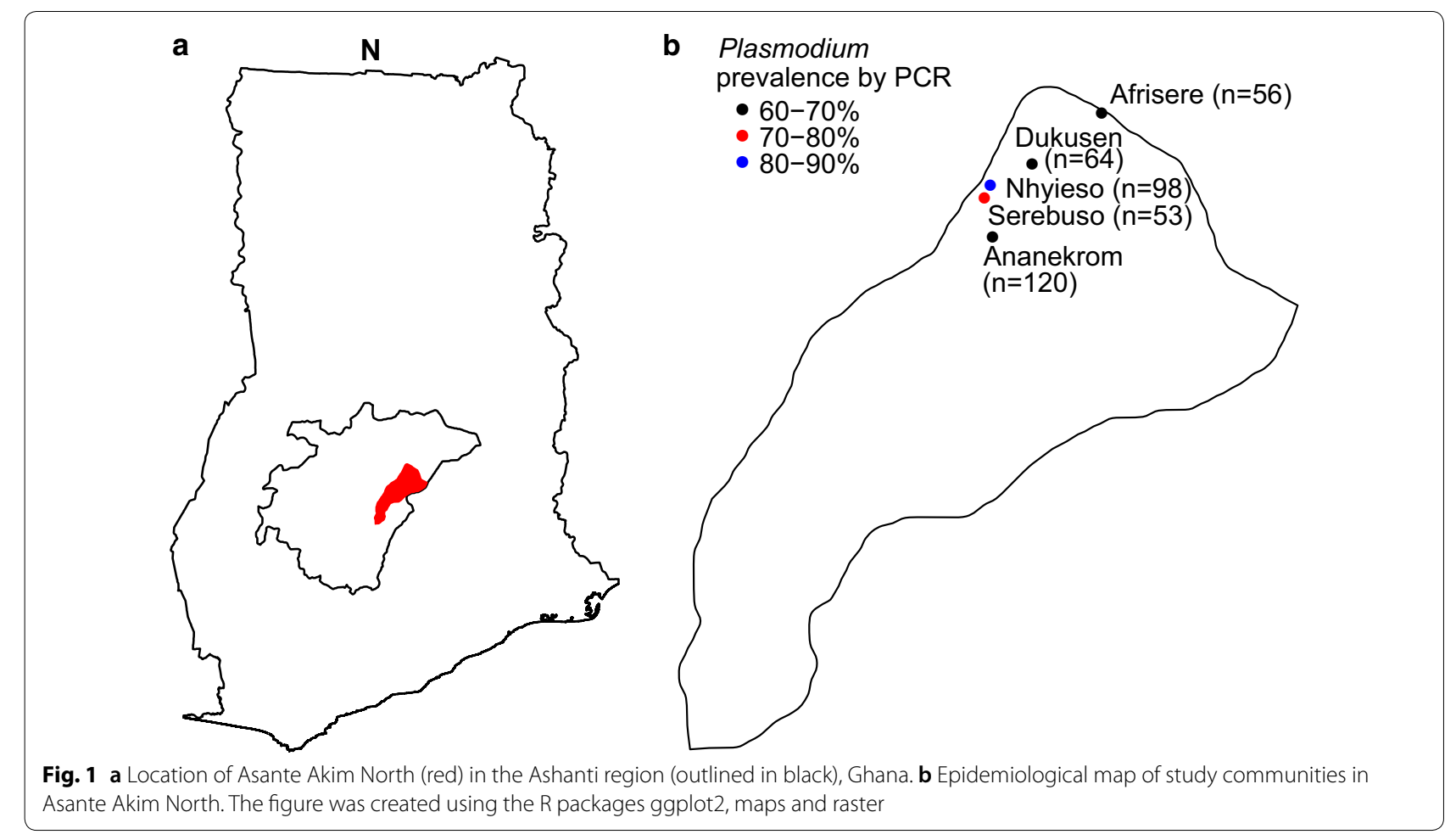


(26/34), respectively. The distribution of Plasmodium infections among different age groups in women and men is demonstrated in Fig. 2a, b.

RDT only detected Plasmodium species in 126 study participants (32\%). Adjusted for age, gender and village, the $C_{t}$ value of the commercial screening PCR was significantly associated with a positive RDT in the whole cohort and in the subgroup with $P$. falciparum monoinfection (Fig. 3a, b). Compared to PCR, sensitivity and specificity of the RDT for detection of Plasmodium infection were $43 \%(122 / 282)$ and $96 \%(102 / 106)$. When interpreting very faint bands $(n=17)$ as positive, sensitivity increased to $48 \%(136 / 282)$ and specificity decreased to $93 \%$ (99/106).

Participants with Plasmodium carriage confirmed by PCR were younger on average (median age, 31 years; IQR, 23-41) compared to participants with negative screening PCR who had a median age of 35 years (IQR, 29-48 years).

Among the parameters measured by blood count, the platelet count was inversely associated with Plasmodium carriage status detected only by PCR in both univariate and multivariate analysis, adjusted for age,
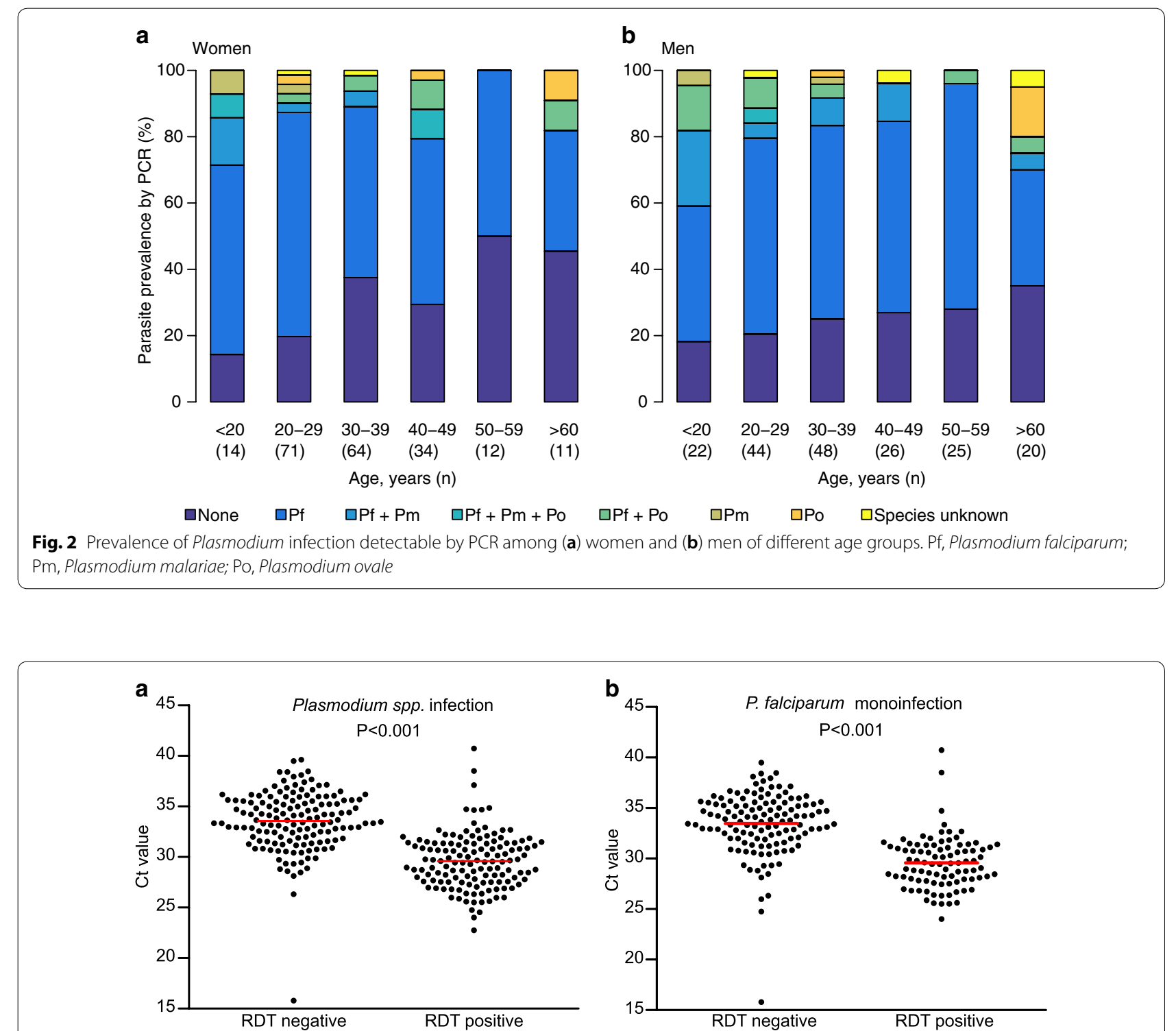

Fig. 3 PCR $C_{t}$ values according to RDT result for a Plasmodium species infection and $\mathbf{b} P$. falciparum monoinfection. Red lines indicate median values. $C_{t}$, cycle threshold; P., Plasmodium; RDT, rapid diagnostic test 
gender and village (Table 2). Haemoglobin and white blood count did not differ between the groups.

\section{Discussion}

The present study conducted in the rainy season in the forest zone of Ghana reveals a very high prevalence of asymptomatic Plasmodium infection, with $73 \%$ of adult residents being PCR-positive for at least one Plasmodium species. In the studied populations, asymptomatic adults represent a relevant reservoir for malaria parasites. Any attempt at malaria eradication therefore must target a wider population and should not only focus on children or individuals with a positive RDT. In endemic regions, surveys should be conducted on a regular basis.

This is the first study on molecular prevalence of asymptomatic Plasmodium infections in adult residents of rural areas in the Ashanti region. A study conducted in 1998 observed a prevalence detected by microscopy of $51 \%$ in the forest area of the Ashanti region with a peak among 8-year-old children and a plateau at about $20 \%$ in adults [14]. The prevalence of adult asymptomatic parasite carriers assessed by PCR was considerably higher in the present study.

In a study including 160 asymptomatic adults and children from the Greater Accra Region in Southern Ghana, the prevalence of asymptomatic parasite carriers based on microscopy was $34 \%$ and $4 \%$ in a high and low malaria transmission area, respectively [15]. However, molecular diagnostic tools for detection of submicroscopic parasitaemia were not performed. In the Upper East region of Ghana, which is considered as a part of the Guinea Savannah Zone, asymptomatic P. falciparum carriage rates detected by PCR among all age-groups were $14 \%$ during the dry season [16]. In the same region, the molecular prevalence of $P$. falciparum infection was $72 \%$ in asymptomatic adults $>19$ years recruited in the rainy season in 2000 [17]. Among adult residents ( $>20$ years) of the Guinea Savannah Zone recruited in 2012-2013, the prevalence of asymptomatic $P$. falciparum infection assessed by combined microscopy and PCR was $64 \%$ and $27 \%$ in the wet and dry seasons, respectively [18].
With 68\%, the prevalence of asymptomatic P. falciparum parasitaemia observed in the present study was, therefore, similar to studies conducted in the rainy season in the Guinea Savannah zone $[17,18]$ and in a rain forest region in Gabon [19]. In a cross-sectional study performed in the Eastern region of Ghana in 2017, the positivity rate among adults 20 years old and above was $14 \%$ by RDT and about $55 \%$ by PCR [20]. Despite the fact that this study included febrile cases also, the observed prevalence was considerably lower than in the present survey.

Subtypes of $P$. ovale have not been widely reported specifically in asymptomatic Ghanaian adults before. Plasmodium ovale was identified in nearly $10 \%$ of the study participants, with similar numbers of individuals positive for P. o. curtisi and P. o. wallikeri. The importance of the identification of $P$. ovale is increasingly recognized, since both subtypes are perceived as relapsing malarial parasites, with $P$. o. curtisi reappearing in shorter time intervals compared to P. o. wallikeri [21]. Asymptomatic infections with $P$. malariae were also common with $8 \%$ in the present study. Comparable to the study from Gabon [19], non-falciparum infections presented mostly as mixed infections with P. falciparum.

Even though the WHO suggests that even faint RDT bands should be interpreted as positive, a recent study concluded that it is possible to interpret faint test bands as malaria negative if the patient does not have risk factors of developing severe malaria [9]. In the present study, $43 \%$ of asymptomatic Plasmodium species carriers were detected by RDT. Even if very faint bands were interpreted as positive, the sensitivity of the RDT was still less than $50 \%$. In a study on MSAT in Zanzibar, antimalarial treatment of RDT-positive individuals did not reduce subsequent malaria incidence, compared with control areas. Only $4 \%$ of parasite carriers were detected by RDT compared to PCR in that study [2]. In the crosssectional study performed in the Eastern region of Ghana, about one out of four malaria cases in individuals aged $\geq 20$ years, which were detected by PCR, were also identified by RDT [20].

Table 2 Predictors of asymptomatic Plasmodium infection in univariate and multivariate analyses

\begin{tabular}{|c|c|c|c|c|c|}
\hline \multirow[t]{2}{*}{ Variable } & \multicolumn{2}{|l|}{ Median (IQR) } & \multirow{2}{*}{$\begin{array}{l}\text { Univariate } \\
\text { P-value }\end{array}$} & \multicolumn{2}{|c|}{ Multivariate $^{a}$} \\
\hline & PCR positive & PCR negative & & $\beta$ & P-value \\
\hline White blood cells $\left(\times 10^{9} / \mathrm{l}\right)$ & $5.5(4.7-6.7)$ & $5.7(4.6-6.9)$ & 0.741 & 0.04 & 0.822 \\
\hline Haemoglobin (mg/l) & $13.1(12.3-14.2)$ & $13.2(12.3-14.3)$ & 0.747 & -0.14 & 0.346 \\
\hline Platelets $\left(\times 10^{9} / \mathrm{l}\right)$ & $225(183-271)$ & $253(196-308)$ & 0.004 & -17.94 & 0.03 \\
\hline
\end{tabular}

For univariate analyses Wilcoxon rank-sum test was used, multivariate analyses were performed using linear regression

a Adjusted for age, gender and village 
The considerable variance in the sensitivity of RDTs among different studies highlights that molecular diagnostic tools are necessary to adequately assess the malaria prevalence and improve MSAT programmes.

\section{Conclusion}

Asymptomatic parasitaemia in adults, including cases with non-falciparum species, constitutes a relevant reservoir for transmission in the Ghanaian forest zone and must be considered in efforts towards elimination of malaria.

\author{
Abbreviations \\ $C_{t}$ : Cycle threshold; IQR: Interquartile range; MSAT: Mass screening and treat- \\ ment; PCR: Polymerase chain reaction; RDT: Rapid diagnostic test; WHO: World \\ Health Organization.
}

\section{Acknowledgements}

The authors acknowledge the staff of the Bernhard Nocht Institute for Tropical Medicine, particularly Stefanie Ruben, Anja Wentzin and Sabine Eberhardt, as well as the staff of the Kumasi Center for Collaborative Research for their enduring and valuable work. They further thank the study participants for their cooperation.

\section{Authors' contributions}

CCR, CDV, MH, ROP and TR were responsible for the study design. MH and ROP collected the blood samples. MH and TR analysed and interpreted the data. ET performed the polymerase chain reaction. MH and TR wrote the first version of the manuscript. CCR, CDV, ROP and ET made edits to the manuscript. All authors read and approved the final manuscript.

\section{Funding}

Open Access funding enabled and organized by Projekt DEAL. The study was supported by a Bayer AG grant. The funding body had no influence on the design of the study, collection, analysis, and interpretation of data, nor on writing the manuscript. TR was supported by a Clinical Leave Fellowship by the German Center for Infection Research (DZIF, 80095CLTHR).

\section{Availability of data and materials}

The datasets used and/or analysed during the current study are available from the corresponding author on reasonable request.

\section{Ethics approval and consent to participate}

The study was approved by the Committee on Human Research, Publication and Ethics at the Kwame Nkrumah University of Science and Technology, Kumasi, Ghana (CHRPE/AP/455/18). Written informed consent was obtained from all participants.

\section{Consent for publication}

Not applicable.

\section{Competing interests}

$\mathrm{CDV}$ received lecture fees from Bayer AG.

\footnotetext{
Author details

1 Division of Infectious Diseases, I. Department of Medicine, University Medical Centre Hamburg-Eppendorf, Hamburg, Germany. ${ }^{2}$ Department of Tropical Medicine, Bernhard Nocht Institute for Tropical Medicine, Hamburg, Germany. ${ }^{3}$ Kumasi Center for Collaborative Research in Tropical Medicine, Kumasi, Ghana. ${ }^{4}$ Department of Medicine, University Medical Centre Hamburg-Eppendorf, Hamburg, Germany. ${ }^{5}$ National Reference Centre for Tropical Pathogens, Bernhard Nocht Institute for Tropical Medicine, Hamburg, Germany. ${ }^{6}$ German Centre for Infection Research (DZIF), Partner Site Hamburg-Lübeck-BorstelRiems, Hamburg, Germany. ${ }^{7}$ Department of Clinical Immunology of Infectious Diseases, Bernhard Nocht Institute for Tropical Medicine, Hamburg, Germany.
} ${ }^{8}$ Present Address: Weizmann Institute of Science, Rehovot, Israel. ${ }^{9}$ Present
Address: NYU Langone Medical Center, New York, NY, USA. ${ }^{10}$ Present Address: Infectious Disease Service, Department of Medicine, Memorial Sloan Kettering Cancer Center, New York, NY, USA.

Received: 3 April 2020 Accepted: 6 October 2020

Published online: 12 October 2020

\section{References}

1. WHO. World malaria report 2019. Geneva: World Health Organization; 2019.

2. Cook J, Xu W, Msellem M, Vonk M, Bergstrom B, Gosling R, et al. Mass screening and treatment on the basis of results of a Plasmodium falciparum-specific rapid diagnostic test did not reduce malaria incidence in Zanzibar. J Infect Dis. 2015;211:1476-83.

3. Bousema T, Okell L, Felger I, Drakeley C. Asymptomatic malaria infections: detectability, transmissibility and public health relevance. Nat Rev Microbiol. 2014;12:833-40.

4. Andrews L, Andersen RF, Webster D, Dunachie S, Walther RM, Bejon $P$, et al. Quantitative real-time polymerase chain reaction for malaria diagnosis and its use in malaria vaccine clinical trials. Am J Trop Med Hyg. 2005;73:191-8.

5. Laishram DD, Sutton PL, Nanda N, Sharma VL, Sobti RC, Carlton JM, et al. The complexities of malaria disease manifestations with a focus on asymptomatic malaria. Malar J. 2012;11:29.

6. de Mast Q, Brouwers J, Syafruddin D, Bousema T, Baidjoe AY, de Groot PG, et al. Is asymptomatic malaria really asymptomatic? Hematological, vascular and inflammatory effects of asymptomatic malaria parasitemia. J Infect. 2015;71:587-96.

7. Krefis AC, Schwarz NG, Kruger A, Fobil J, Nkrumah B, Acquah S, et al. Modeling the relationship between precipitation and malaria incidence in children from a holoendemic area in Ghana. Am J Trop Med Hyg. 2011;84:285-91.

8. WHO. Malaria rapid diagnostic test performance: results of WHO product testing of malaria RDTs: round 8 (2016-2018). Geneva: World Health Organization; 2018

9. Makuuchi R, Jere S, Hasejima N, Chigeda T, Gausi J. The correlation between malaria RDT (Paracheck pf.(R)) faint test bands and microscopy in the diagnosis of malaria in Malawi. BMC Infect Dis. 2017;17:317.

10. Frickmann H, Hinz R, Rojak S, Bonow I, Ruben S, Wegner C, et al. Evaluation of automated loop-mediated amplification (LAMP) for routine malaria detection in blood samples of German travellers-a cross-sectional study. Travel Med Infect Dis. 2018;24:25-30.

11. Hagen RM, Hinz R, Tannich E, Frickmann H. Comparison of two real-time PCR assays for the detection of malaria parasites from hemolytic blood samples. Eur J Microbiol Immunol. 2015;5:159-63.

12. Frickmann $\mathrm{H}$, Wegner $\mathrm{C}$, Ruben S, Loderstadt U, Tannich E. A comparison of two PCR protocols for the differentiation of Plasmodium ovale species and implications for clinical management in travellers returning to Germany: a 10-year cross-sectional study. Malar J. 2019;18:272.

13. Ablordey AS, Vandelannoote K, Frimpong IA, Ahortor EK, Amissah NA, Eddyani $M$, et al. Whole genome comparisons suggest random distribution of Mycobacterium ulcerans genotypes in a Buruli ulcer endemic region of Ghana. PLoS Negl Trop Dis. 2015;9:e0003681.

14. Browne EN, Frimpong E, Sievertsen J, Hagen J, Hamelmann C, Dietz K, et al. Malariometric update for the rainforest and savanna of Ashanti region, Ghana. Ann Trop Med Parasitol. 2000;94:15-22.

15. Abukari Z, Okonu R, Nyarko SB, Lo AC, Dieng CC, Salifu SP, et al. The diversity, multiplicity of infection and population structure of $P$. falciparum parasites circulating in asymptomatic carriers living in high and low malaria transmission settings of Ghana. Genes (Basel). 2019;10:434.

16. Atelu GR, Duah NO, Wilson MD. Carriage of sub-microscopic sexual and asexual Plasmodium falciparum stages in the dry season at Navrongo, Ghana. Ghana Med J. 2016;50:220-7.

17. Owusu-Agyei S, Smith T, Beck HP, Amenga-Etego L, Felger I. Molecular epidemiology of Plasmodium falciparum infections among asymptomatic inhabitants of a holoendemic malarious area in northern Ghana. Trop Med Int Health. 2002;7:421-8.

18. Tiedje KE, Oduro AR, Agongo G, Anyorigiya T, Azongo D, Awine T, et al. Seasonal variation in the epidemiology of asymptomatic Plasmodium 
falciparum infections across two catchment areas in Bongo District, Ghana. Am J Trop Med Hyg. 2017;97:199-21212.

19. Woldearegai TG, Lalremruata A, Nguyen TT, Gmeiner M, Veletzky L, Tazemda-Kuitsouc GB, et al. Characterization of Plasmodium infections among inhabitants of rural areas in Gabon. Sci Rep. 2019;9:9784.

20. Amoah LE, Donu D, Abuaku B, Ahorlu C, Arhinful D, Afari E, et al. Probing the composition of Plasmodium species contained in malaria infections in the Eastern region of Ghana. BMC Public Health. 2019;19:1617.

21. Groger M, Veletzky L, Lalremruata A, Cattaneo C, Mischlinger J, Manego Zoleko $\mathrm{R}$, et al. Prospective clinical and molecular evaluation of potential
Plasmodium ovale curtisi and wallikeri relapses in a high-transmission setting. Clin Infect Dis. 2019;69:2119-266.

\section{Publisher's Note}

Springer Nature remains neutral with regard to jurisdictional claims in published maps and institutional affiliations.
Ready to submit your research? Choose BMC and benefit from:

- fast, convenient online submission

- thorough peer review by experienced researchers in your field

- rapid publication on acceptance

- support for research data, including large and complex data types

- gold Open Access which fosters wider collaboration and increased citations

- maximum visibility for your research: over $100 \mathrm{M}$ website views per year

At BMC, research is always in progress.

Learn more biomedcentral.com/submissions 